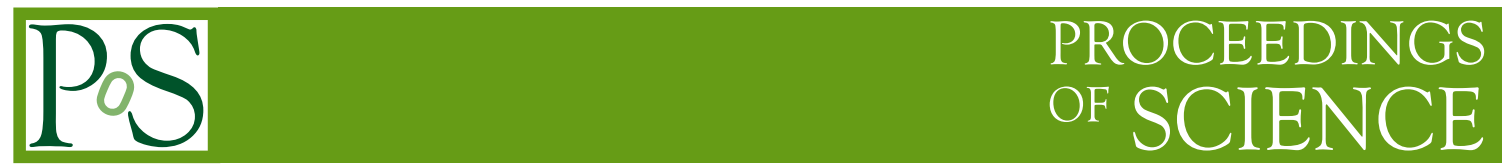

\title{
Baryon resonances at large number of colors
}

\section{Victor Petrov*}

Petersburg Nuclear Physics Institute, Gatchina 188300, St. Petersburg, RussiaPNPI

E-mail: Victor.Petrovathd.pnpi.spb.ru

We suggest a new picture of baryon resonances below $2 \mathrm{GeV}$ based on the quark-soliton model in the limit of large number of colors which is able to describe all known resonances in this region

XV International Conference on Hadron Spectroscopy 4-8 November 2013

Nara, Japan

${ }^{*}$ Speaker. 


\section{Foreword}

Present section of our conference is devoted to the memory of Dmitry Diakonov, my friend and co-author. We worked with him for more than 30 years.

Mitya entered the Theory Division of Petersburg nuclear Physics Institute in 1972. A.A. Anselm and V.N.Gribov were his scientific advisors. His first papers were devoted to subtle ques-

tions of the quantum field theory: spontaneous breakdown of chiral invariance in perturbation theory, radiative corrections to Coleman-Weinberg effective potential and to the Weinberg angle in the Standard model and GUT. Quantum field theory remained Mitya's love for all his life.

He became known in 1978 for his papers on hard processes in QCD [1] where physics of

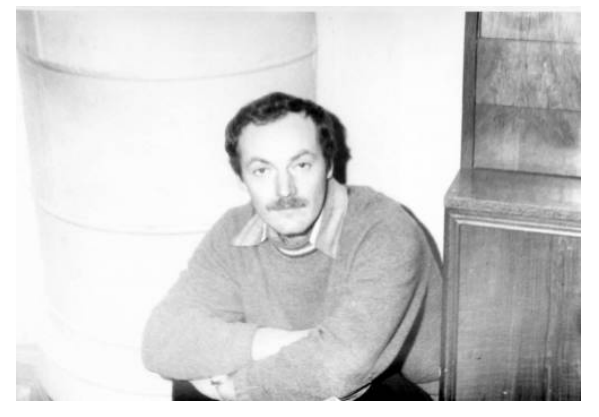

Dmitri Diakonov, 1977

Drell-Yan process was explained and famous DDT-formula was derived. However, even that time he feels that perturbative theory is unable to give answers to the most interesting questions in QCD.

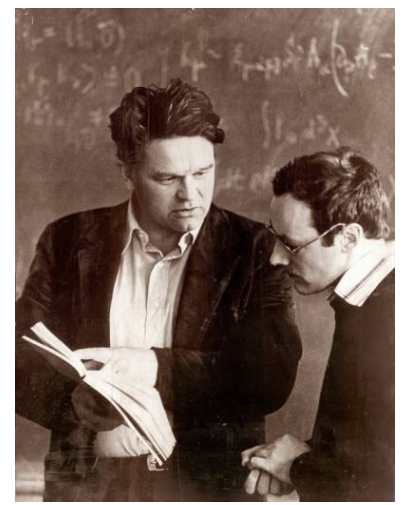

Dmitri Diakonov (right) and Ludwig Faddeev (left), 1981
In 1980 we started a long-standing program of the investigation of non-perturbative phenomena in QCD. Adopting the hypothesis that instantons are the only large fluctuation we developed a theory which is known now under the name "instanton liquid model" [2]. The model appeared to be surprisingly successful: it contains most of the features of QCD: spontaneous breakdown of chiral symmetry, solution of $U(1)$ problem, constituent quarks and massless pions. It reflects QCD not only qualitatively but also quantitatively: its predictions tend to coincide with the data without any fitting parameter.

The next step was the construction of the chiral theory of nucleon following from instanton model [3]. Mitya spent quite

a time investigating properties of the baryons in this model. This experience led us in 1997 to the prediction of narrow exotic antidecuplet of pentaquarks. This topic became very important for Mitya: he made his best to convince experimentalists to check the prediction and returned many times to pentaquarks in his papers. Unfortunately, the final answer is still unclear. 


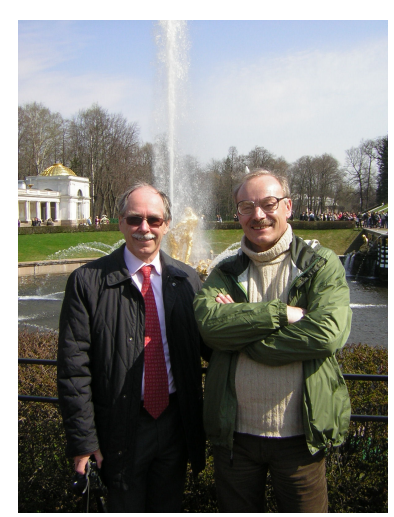

Discussing confinement with G.t'Hooft, 2006

Mitya has a lively and charming personality. He played piano and guitar, was fond on the hiking in high mountains. In our small group he played simultaneously the role of motorman and ideas generator.

My talk is based on the one of the last papers with Mitya which was published already after his death [6].
Instanton model has an evident drawback: it does not account for quark confinement. Mitya saw it very clearly and published a number of papers trying to realize a nature of this mysterious phenomenon (see, e.g., [5]). The area of his interests in quantum field theory was very wide. For example, last years he became enthusiastic about quantum gravity and published few papers on the subject.

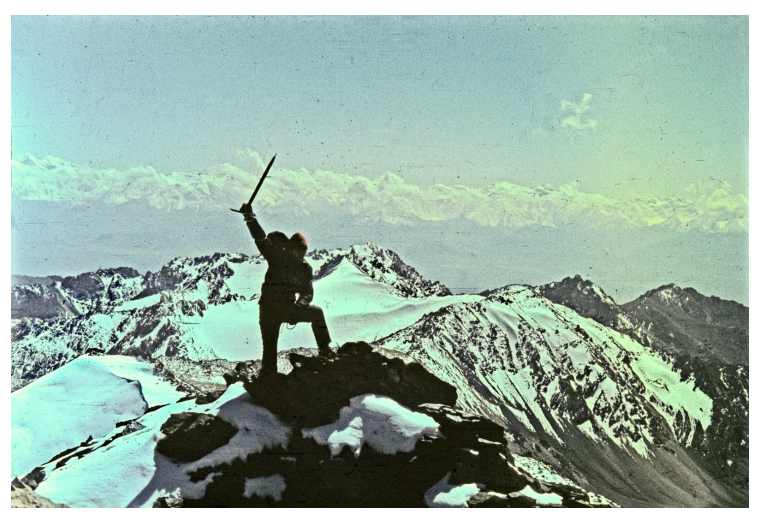

In high mountains, Pamir, 1982.

\section{Relation of Quark model and soliton models of baryons at large $N_{c}$}

There are two popular points of view on baryon resonances. The first is the old good quark model with all baryons made of three constituent bounded by some colorless force. The second suggests that all baryons can be obtained as solitons of some meson Lagrangian. In fact, the second approach can be proved rigorously in QCD in the limit of large number of colors $N_{c}$ [7]. From the other hand, the quark model has no other foundations except the evident success in describing of the date (for review see, e.g., [8]).

A bridge between two approaches is provided by so-called chiral Quark-Soliton model ( $\chi \mathrm{QSM})$ [3]. In the framework of this model it is assumed that at large $N_{c}$ QCD is reduced to effective low energy theory with degrees of freedom which are constituent quarks and colorless meson fields. This theory is essentially semiclassical: interaction of mesons is suppressed by $N_{c}$. The spectrum of mesons can be read off the effective lagrangian, baryons appear as solitons which are the bound states of $N_{c}$ quarks in the self-consistent meson field.

The introduction of separate degrees of freedom for consituent quarks and mesons should be justified: indeed, as mesons ultimately consists of quarks again, so this can be a double-counting. Let us outline the derivation of such a low energy effective theory from QCD. At the first stage one has to integrate out gluons, the theory then reduces to the theory of quarks only. Owing to the expected spontaneous chiral symmetry breaking these constituent quarks are massive, their interaction is described by some many-fermion non-local terms. This interaction can be rewritten 
in colorless channels and at large $N_{c}$ bosonized by means of some auxiliary meson fields. Meson field introduced this way do not lead to double counting and the corresponding effective theory is self consistent.

This programme was carried out in the framework of the instanton liquid picture of the QCD vacuum [2]. Here the integration in all gluon degrees of freedom are restricted to instantons and small fluctuations around them. One can derive the corresponding low energy theory, it consists of the constituent quarks with a momentum dependent mass $M(p)$ and massless pions interacting by means of the simple effective Lagrangian. The model of baryons based on this idea is working rather good describing all known properties of low lying baryons [3] except one but, maybe, the most important - the model does not contain the quark confinement (it is not reproduced in the instanton liquid picture). However, this is only a particular realization of the effective theory under discussion, in fact, it is easy to suggest phenomenological generalizations which have confinement built-in.

Quark model at large $N_{c}$ and the theory of baryon-soliton are extreme cases of the mean-field effective theory. Quark model represents the non-relativistic approximation to the theory. In this case one can neglect quark-aniquark fluctuations (which are not suppressed in the limit of $N_{c} \rightarrow \infty$ and are present in any relativistic field theory) and view baryon as consisting of precisely $N_{c}$ quarks. Neglecting also the retarding of the quark-quark interaction we will arrive at the potential quark model which one can consider in the mean field approximation at large $N_{c}$.

From the other hand, integrating in quark field we obtain the meson Lagrangian where baryon should appear as soliton. Usually, meson lagrangian is constructed as expansion in the gradients of the meson field assuming that meson mean field is slowly varying. It can be seen that this corresponds to the baryon state in which the contribution of Fock components with the large number of quark-antiquark pairs is dominating and valence quarks are ultra-relativistic. Hence, this case is opposite to the case of quark model.

Information about quark degrees of freedom is lost in the soliton Skyrme-like models. For this reason there is no direct way to describe baryon resonances in such models - these models can be applied only to the ground state baryons (lowest baryon octet and decuplet). One has to consider scattering amplitudes in order to discover baryon resonances as poles of amplitudes in the complex plane. $\chi$ QSM allows one to construct baryon states directly, as the eigenfunctions of some Hamiltonian.

Quark model can be also considered at large number of colors. In this case one can also apply the mean field approximation. The main difference with soliton model or $\chi$ QSM (besides the fact that constituent quarks are supposed to be non-relativistic) is the symmetry of the field. Both in the Skyrme model and in $\chi$ QSM the mean field has a so-called hedgehog symmetry, while quark model starts from the spherically symmetrical $S U(6)$-invariant mean field.

The states of quarks in such a field are characterized by the conserved quantity — grand spin $\mathbf{K}=\mathbf{J}+\mathbf{T}$ where $\mathbf{J}$ is a total angular moment of the quark and $\mathbf{T}$ is its isospin. Owing to the symmetry of mean field there are completely different set of levels for $u, d$-quarks and $s$-quarks. One has to find discrete levels in the mean field and distribute $N_{c}$ valence quarks in these levels. The ground state baryons correspond to all quarks sitting in one, ground level, excited baryons appear when we place one or more quarks to excited levels, etc. The $S U(3)_{F} \otimes O(3)$ symmetry broken by the symmetry of the mean field is restored when one consider rotational states of the baryons. 


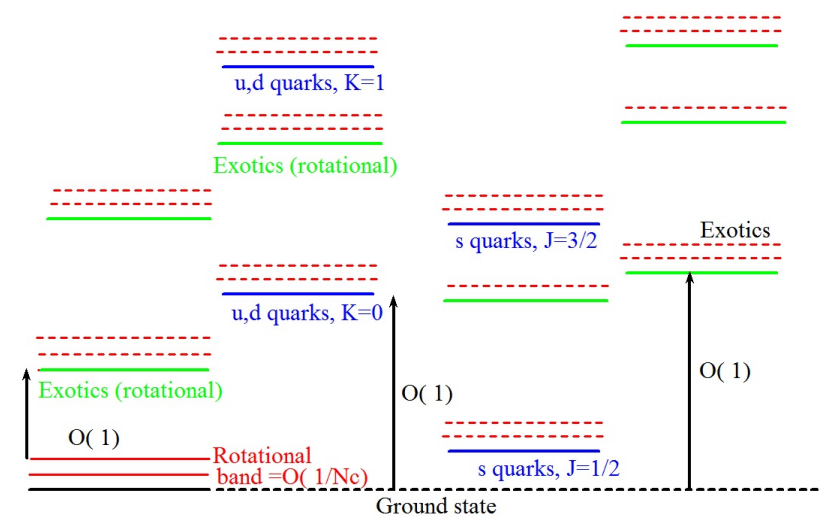

Figure 1: Baryon levels at $N_{c} \rightarrow \infty$

Every discrete level gives rise to the finite rotational band of the baryon resonances with the splitting which is of order $O\left(1 / N_{c}\right)$ while the distance between levels is $O(1)$. Only few lowest states of this band are made of 3 quarks; all remaining states are exotic.

\section{Baryon resonances in $\chi \mathrm{QSM}$}

In paper [6] we construct the quantum theory of rotations in $\chi \mathrm{QSM}$. The general formula for the mass of the baryon resonance lying on the rotational band is of the form:

$\mathscr{M}=\mathscr{M}_{0}+\Delta \varepsilon_{\text {lev }}+\frac{1}{2 I_{1}}\left[a_{K} J(J+1)+\left(1-a_{K}\right) \widetilde{T}(\widetilde{T}+1)-a_{K}\left(1-a_{K}\right) K(K+1)\right]+\frac{(1+X)(2+3 \widetilde{Y})}{2 I_{2}}$

Here $\Delta \varepsilon_{l e v}$ are energies of discrete levels in the mean field, $K$ is its grand spin. $J$ is the spin of resonance, $\widetilde{T}$ and $\widetilde{Y}$ are internal quantum numbers characterizing the soliton in the rotating frame. $\widetilde{T}$ is isospin and $\widetilde{Y}$ is hypercharge. The last one should obey famous Witten quantization rule $\widetilde{Y}=N_{c} / 3$ while possible 'isospins' $\widetilde{T}$ should be found from vector quantization rule $\widetilde{\mathbf{K}}=\widetilde{\mathbf{T}}+\mathbf{J}$. These quantization rules restrict possible baryon multiplets. $I_{1}$ and $I_{2}$ are moments of inertia of baryon soliton which are the same for all resonances, $a_{K}$ is a numeric constant determined by the properties of the given level (for details, see [6]).

At last, $X$ is the exoticness of the state which is defined as a minimal number of the quarkantiquark pairs needed to create a resonance with such quantum numbers. For ordinary baryons $X=0$.

Confronting formula (3.1) to the masses of baryon resonances one can find the following interpretation of resonances as rotational states o baryon soliton in the $\chi$ QSM . It is important that all baryon resonances can fit to this scheme. All resonances can be interpreted as 1-quark excitations in the mean field. There no extra or missing states.

Formula (3.1) gives then relation between masses of baryon resonances lying in the same rotational band. There are also some relations for mass splittings in the multiplets, coupling constants 


\begin{tabular}{|c|c|c|c|}
\hline quark levels & rotational bands & $\left(I_{1}\right)^{-1}, \mathrm{MeV}$ & $\tilde{a}_{K}$ \\
\hline $\begin{array}{l}K^{P}=0^{+}, \text {ground } \\
\text { state }\end{array}$ & $\left(\mathbf{8}, 1 / 2^{+}, 1152\right) \quad\left(\mathbf{1 0}, 3 / 2^{+}, 1382\right)$ & 153 & \\
\hline $0^{+} \rightarrow 0^{+} \quad 482 \mathrm{MeV}$ & $\left(\mathbf{8}, 1 / 2^{+}, 1608\right) \quad\left(\mathbf{1 0}, 3 / 2^{+}, 1732\right)$ & 83 & \\
\hline $0^{+} \rightarrow 2^{+} \quad 722 \mathrm{MeV}$ & $\begin{array}{ll}\left(\mathbf{8}, 3 / 2^{+}, 1865\right) & \left(\mathbf{8}, 5 / 2^{+}, 1873\right) \\
\left(\mathbf{1 0}, 3 / 2^{+}, 2087\right) & \left(\mathbf{1 0}, 5 / 2^{+}, 2071\right) \\
\left(\mathbf{1 0}, 7 / 2^{+}, 2038\right) & \end{array}$ & 131 & -0.050 \\
\hline $0^{+} \rightarrow 1^{+} \sim 780 \mathrm{MeV}$ & $\begin{array}{ll}N\left(1 / 2^{+}, 1710\right) & N\left(3 / 2^{+}, 1900\right) \\
\Delta\left(1 / 2^{+}, 1910\right) & \Delta\left(3 / 2^{+}, \sim 1945\right) ? \\
\Delta\left(5 / 2^{+}, 2000\right) & \end{array}$ & & \\
\hline $0^{+} \rightarrow 1^{-} \quad 468 \mathrm{MeV}$ & $\begin{array}{ll}\left.\mathbf{( 8}, 1 / 2^{-}, 1592\right) & \left(\mathbf{8}, 3 / 2^{-}, 1673\right) \\
\left(\mathbf{1 0}, 1 / 2^{-}, 1758\right) & \left(\mathbf{1 0}, 3 / 2^{-}, 1850\right)\end{array}$ & 171 & 0.336 \\
\hline $0^{+} \rightarrow 0^{-} \quad 563 \mathrm{MeV}$ & $\left(\mathbf{8}, 1 / 2^{-}, 1716\right)$ & 155 (fit) & \\
\hline $0^{+} \rightarrow 2^{-} \quad 730 \mathrm{MeV}$ & $\left(\mathbf{8}, 3 / 2^{-}, 1896\right) \quad\left(\mathbf{8}, 5 / 2^{-}, 1801\right)$ & 155(fit) & -0.244 \\
\hline $0^{+} \rightarrow \frac{1}{2}^{-} \quad 254 \mathrm{MeV}$ & $\left(\mathbf{1}, 1 / 2^{-}, 1405\right)$ & & \\
\hline $0^{+} \rightarrow \frac{3}{2}^{-} \quad 379 \mathrm{MeV}$ & $\left(\mathbf{1}, 1 / 2^{-}, 1520\right)$ & & \\
\hline
\end{tabular}

Table 1: Interpretation of all baryon resonances below $2 \mathrm{GeV}$, as rotational excitations on top of intrinsic quark states.

for interaction with mesons etc. These relation are model independent and tend to coincide with data. If, in addition, the concrete meson Lagrangian is fixed, all properties of the baryon resonances can be calculated (see Ref.[6]) .

\section{Conclusions}

We suggested the self-consistent description of the baryon resonances below $2 \mathrm{GeV}$ based on the limit $N_{c} \rightarrow \infty$. This description generalizes results of both quark model and soliton approach to baryons which are the limiting cases of our approach. All known low-lying baryon resonances naturally fit this scheme and there are no missing states. It is possible to derive a number of new, model-independent relations for properties of resonances which coincide with experimental data.

Acknowledgements The author acknowledges organizers and especially Atsushi Hosaka for the invitation and their support which make it possible to attend the conference. Special thanks are due to Hyun-Chul Kim for fruitful discussions and his hospitality in INHA University where the part of this work was done. Author is also grateful to A.Vladimirov, the co-author of the paper [6] on which this contribution is based.

\section{References}

[1] Yuri L. Dokshitzer, Dmitri Diakonov, S.I. Troian, Hard Processes in Quantum Chromodynamics, Phys.Rept. 58 (1980) 269-395 
[2] Dmitri Diakonov, V.Yu. Petrov, Instanton Based Vacuum from Feynman Variational Principle, Nucl.Phys. B 245 (1984) 259; A Theory of Light Quarks in the Instanton Vacuum, Nucl.Phys. B 272 (1986) 457.

[3] Dmitri Diakonov, V.Yu. Petrov, P.V. Pobylitsa A Chiral Theory of Nucleons, Nucl.Phys. B 306 (1988) 809

[4] Dmitri Diakonov,Victor Petrov, Maxim V. Polyakov, Exotic anti-decuplet of baryons: Prediction from chiral solitons, Z.Phys. A 359 (1997) 305-314

[5] Dmitri Diakonov, Victor Petrov Confining ensemble of dyons Phys.Rev. D 76 (2007) 056001

[6] Dmitri Diakonov, Victor Petrov, Alexey A. Vladimirov, A theory of baryon resonances at large $N_{c}$, Phys.Rev. D 88 (2013) 074030

[7] E. Witten, Baryons in the 1/N Expansion, Nucl. Phys. B 160 (1979) 57; G.S. Adkins, C.R. Nappi, and E. Witten, Nucl. Phys., B 228 (1983) 552.

[8] Glozman and D. O. Riska, Phys. Rep. 268 (1996), 263; S.Capstick and W. Roberts, Prog. Part. Nucl. Phys. 45 (2000), 241 